\title{
MEJORAMIENTO GENÉTICO DEL FRIJOL COMÚN MEDIANTE ENFOQUES PARTICIPATIVOS EN HONDURAS1
}

\author{
Juan Carlos Rosas², Omar Gallardo², José Jiménez ${ }^{2}$
}

\begin{abstract}
RESUMEN
Mejoramiento genético del frijol común mediante enfoques participativos en Honduras. El presente trabajo presenta resultados de la aplicación de metodologías participativas en el mejoramiento genético del frijol en dos regiones de Honduras. Se describen las metodologías y se presentan los resultados de las actividades de evaluación y selección, desde generaciones tempranas en poblaciones segregantes, hasta los ensayos de comparación del comportamiento de líneas avanzadas seleccionadas por métodos convencionales y participativos, y los ensayos de validación de líneas promisorias seleccionadas para estimar su potencial de adopción como variedad mejorada. Se mencionan los criterios de evaluación y las decisiones para la selección optadas por los agricultores en la selección de líneas y variedades. Se discuten los resultados tratando de explicarlos según los principios y prácticas fundamentales en el mejoramiento de las plantas cultivadas. Finalmente, se identifican los beneficios del mejoramiento participativo con los agricultores, incluyendo aquellos relacionados con la adquisición de conocimientos y destrezas, y el incremento de la capacidad individual y colectiva en la toma de decisiones; así como las ventajas para los programas de mejoramiento bajo estos enfoques participativos.
\end{abstract}

\begin{abstract}
Common bean through a participatory approach in Honduras. This work presents the results of the application of participatory methods for the genetic improvement of beans in two regions of Honduras. The methodology and results obtained from the evaluation and selection activities, from early generation to advanced trials compare the lines selected by conventional and participatory methods, and the trials to estimate the potential of adoption of promising lines, are shown and described. Evaluation and selection criteria used by farmers to choose promising lines and varieties are mentioned. The results are discussed, trying to explain them according to the basic plant breeding principles and practices for the improvement of cultivated plants. Finally, the benefits of participatory plant breeding for farmers are identified, including those that are related to knowledge and skill acquisitions, and the increase on individual and collective capacity to take decisions; as well as the advantages for breeding programs under these participatory approaches.
\end{abstract}

\section{INTRODUCCIÓN}

En el año 2000, Honduras produjo 85,108 t de frijol (Phaseolus vulgaris L.) en 121,121ha, con un rendimiento promedio de $702 \mathrm{~kg} / \mathrm{ha}$ (Cotty et al. 2001). El consumo per cápita nacional es de $23 \mathrm{~kg}$, pero este puede llegar a ser hasta el doble en las zonas rurales. La mayoría de los productores de frijol tienen acceso limi- tado a los mercados debido a la falta de transporte, caminos inadecuados y distancias alejadas a las ciudades principales; por estas razones, la mayor parte de la producción es vendida a intermediarios en las fincas. El precio promedio en la finca fue de $\$ 33.60$ por quintal (46 kg), pero los precios fluctúan considerablemente debido a cosechas estacionarias. Aún así, el frijol es el sétimo cultivo en importancia económica en Honduras.

1 Recibido para publicación el 21 de enero del 2003. Trabajo conducido en colaboración con agricultores de las regiones de Yorito y el Lago Yojoa, Honduras.

2 Fitomejorador, Programa de Investigaciones en Frijol, Escuela Agricola Panamericana, Zamorano, Apartado Postal 93, Tegucigalpa, Honduras (jcrosas@zamorano.edu ); e investigadores del Proyecto IPCA, Plaza del Caribe No. 207, La Ceiba, Honduras (proyectoipca@caribe.hn ). 
La mayoría del frijol es producido en pequeñas fincas mayormente ubicadas en terrenos de laderas y suelos marginales. Los agricultores usan bajos insumos y sólo algunas fincas son mecanizadas o irrigadas. Debido a su precocidad, buena adaptación y excelente calidad de grano, las variedades criollas y las "acriolladas" (variedades mejoradas antiguas, p.e. Desarrural y Danlí 46), son utilizadas extensivamente en Honduras. Sin embargo, debido a la alta susceptibilidad de la mayoría de estas variedades, cerca del $40 \%$ de productores de frijol usan variedades mejoradas resistentes a enfermedades. Para conservar la biodiversidad representada por las variedades criollas, los programas convencionales requieren de enfoques de mejoramiento que simultáneamente permitan ampliar la base genética de estas variedades y contribuir a un mayor rendimiento y mejor estabilidad. Algunas alternativas recientes se basan en la implementación de enfoques más participativos, para facilitar la intervención de los agricultores para complementar y agilizar los esfuerzos de los programas de mejoramiento convencionales.

En la última década, los enfoques utilizados por los programas de mejoramiento han sido enfatizados en la generación de cultivares con una base genética más amplia y mayor adaptación regional. Para ello, fuentes diversas de germoplasma de las razas andinas y mesoamericanas se emplean en los programas más relevantes en la región de Centroamérica y El Caribe (Rosas et al. 2000). Actualmente, algunas variedades y germoplasma mejoradas poseen una base genética adecuada que les confiere mayor adaptación y potencial de rendimiento, así como mejor resistencia a enfermedades y factores abióticos, que las variedades criollas. Lamentablemente, los beneficios derivados del empleo de variedades mejoradas están frecuentemente limitados por sistemas deficientes de diseminación y el reducido acceso de los agricultores a semilla de alta calidad. En otros casos, las variedades mejoradas no son adoptadas debido a su inferior calidad de grano con respecto a las criollas, o la falta de adaptación a los sistemas de producción de bajos insumos usados por los pequeños productores.

La aplicación de metodologías de fitomejoramiento participativo (FP) ha sido sugerida como una valiosa alternativa para facilitar el acceso a los agricultores a materiales mejorados con una base genética más amplia; así como, la aplicación de procesos de selección y validación para el desarrollo de cultivares más productivos y estables, adaptados a sus condiciones agro-ecológicas específicas y con mejor aceptación de consumo y comercial. Al seguir procesos de FP, fitomejoradores y agricultores desarrollan cultivares a partir de poblaciones segregantes bajo ambientes metas específicos. Para algunos investigadores, las alternativas de FP de- ben aplicarse cuando los enfoques convencionales resultan incapaces de proveer a los agricultores con variedades adecuadas para sus condiciones específicas.

En este artículo se presentan y discuten parte de los enfoques metodológicos, resultados y experiencias derivadas de la aplicación de alternativas de FP por grupos de agricultores de Honduras, para mejorar la adaptación agronómica, arquitectura de planta y rendimiento de algunas variedades criollas. Se describen las alternativas de FP y los resultados de la selección aplicada desde generaciones tempranas a avanzadas son presentados, así como los resultados de ensayos de adaptación y rendimiento conducidos para comparar las líneas desarrolladas por alternativas de FP vs. convencional. La mayoría de la información en este documento fue obtenida de las actividades de FP de frijol conducidas en Honduras, como parte de un proyecto financiado a través de donaciones otorgadas por el Programa de Investigación Participativa y Análisis de Género (PRGA) del CGIAR. Estas actividades fueron conducidas en colaboración con el Proyecto de Investigación Participativa para Centroamérica (IPCA) de la Universidad de Guelph, Canadá.

\section{MATERIALES Y MÉTODOS}

Las actividades de FP fueron conducidas con la activa participación de agricultores de cuatro comunidades de Yorito, departamento de Yoro, y dos comunidades de los Departamentos de Comayagua y Santa Bárbara, localizadas en los alrededores del Lago Yojoa, Honduras. Los agricultores participantes están organizados bajo el sistema de comité de investigación agrícola local (CIAL), descritos por Ahsby et al. (1995) y Humphries et al. (2000); estos agricultores poseen experiencia previa en investigación participativa.

\section{Evaluación de poblaciones de mejoramiento (segre- gantes)}

Para validar procesos y productos de FP, se emplearon tres alternativas: selección del agricultor a partir de generaciones tempranas (FP-1); selección del agricultor a partir de generaciones avanzadas (FP-2) y selección por el fitomejorador o convencional (FC). Para evaluar estas alternativas, se desarrollaron poblaciones de mejoramiento mediante cruzamientos de variedades de los agricultores con líneas o variedades mejoradas con caracteres deseables como arquitectura erecta, resistencia a enfermedades y buen potencial de rendimiento. Varias poblaciones fueron desarrolladas cruzando las variedades locales "Concha Rosada" de 
Yorito y "Madura Parejo" y "Vaina Blanca" del Lago Yojoa, con líneas élites usadas como padres donantes de caracteres deseables para su mejoramiento. Estas poblaciones mejoradas fueron usadas para evaluar las alternativas de FP indicadas arriba. En esta publicación, se presentan los resultados de las actividades de evaluación y selección conducidas en las comunidades de la región de Yorito, para ilustrar el progreso en la aplicación de metodologías de FP en Honduras.

Los caracteres a ser mejorados mediante cruzamiento y selección fueron previamente identificados por los agricultores participantes en talleres de planificación; estos caracteres fueron arquitectura erecta, resistencia a enfermedades (principalmente antracnosis y roya) y rendimiento. En la alternativa FP-1, los agricultores empezaron a aplicar criterios de selección en cuatro poblaciones segregantes F3, PPB 11 y PPB 12 sembradas en Junio 2000 y PPB 15 y PPB 16 sembrada en la siguiente época, en noviembre 2000. Estas poblaciones F3 fueron sembradas en la comunidad de Mina Honda, empleándose un lote accesible a los agricultores participantes, considerado como el "centro de selección participativa". Las parcelas fueron organizadas siguiendo diseños para evaluar poblaciones de mejoramiento y líneas avanzadas; estos lotes fueron manejados por los agricultores usando sus propias prácticas de siembra y manejo del cultivo. Para las evaluaciones de poblaciones segregantes (F3-F6), las familias fueron distribuidas en parcelas de surcos sencillos con una población entre 30 y 50 plantas por familia, según la disponibilidad de semilla; surcos de la variedad criolla fueron sembrados como testigos cada 10 surcos de familias. Las aplicaciones de fertilizantes fueron similares a las usadas por los agricultores, equivalentes a 65 $\mathrm{kg} / \mathrm{ha}$ de 18-46-0 a los ocho días después de emergencia (DDE) y $65 \mathrm{~kg} / \mathrm{ha}$ de urea a los 30 DDE.

Por consenso y experiencia previa de los agricultores, las evaluaciones de las familias F3 fueron realizadas en tres etapas: valor agronómico (incluyendo arquitectura de planta, reacción a enfermedades, días a madurez y carga reproductiva) en la etapa de llenado de grano; rendimiento de grano a la cosecha y valor comercial del grano (color, tamaño y forma) a la poscosecha. Las evaluaciones fueron hechas con base al comportamiento promedio de las familias, pero la selección fue basada en las mejores plantas de las mejores familias en la etapa de llenado de grano, y compuestos de familias a la cosecha y poscosecha. Después que los resultados de las evaluaciones de las familias F3 fueron discutidos, cada grupo decidió sembrar y continuar la selección de las familias F4 y las generaciones siguientes en sus propias comunidades. Es importante mencionar, que el número y las familias F3 seleccionadas no fueron las mismas en cada comunidad, aunque algunas fueron seleccionadas por más de una comunidad. Las familias F4 fueron sembradas usando parcelas de cuatro surcos de $5 \mathrm{~m}$, incluyendo un promedio de 200 plantas por familia. En la generación F5, se condujeron pruebas de progenie de las plantas individuales F4 seleccionadas. Las plantas seleccionadas de las familias F5 fueron cosechadas en compuesto, y la semilla F6 de estas plantas fue usada en el ensayo comparativo de líneas avanzadas conducido en cinco localidades en la postrera del 2001, el cual se describe más adelante.

En la alternativa FP-2, las plantas fueron avanzadas sin selección desde la F2 hasta la F7 por el método de descendencia de semilla individual (DSI), descrito por Brimm (1966).

Teóricamente, el método de DSI ayuda a mantener la varianza genética de la F2 hasta que las familias se convierten en líneas altamente homocigotas (F6 en adelante), después de varias generaciones de autofecundación. La DSI ha probado ser efectiva en el mejoramiento de caracteres cuantitativos, de baja heredabilidad, en cultivos autógamos. En el presente estudio, las familias DSI-F7 fueron evaluadas por los agricultores en surcos individuales alternados por surcos testigos de la variedad criolla, como se describió en la alternativa FP-1. El método DSI evitaría la evaluación intensiva por varias generaciones, y podría ser útil en comunidades en que la experiencia, mano de obra y otros recursos se encuentran limitados. Al usar el método de DSI, los agricultores serían capaces de mejorar caracteres cuantitativos, así como cualitativos, usando ensayos replicados y seleccionando por el comportamiento promedio de las familias en vez de plantas individuales. Las líneas F7 desarrolladas por este método fueron evaluadas por los grupos de agricultores, durante la siembra de primera (mayo-agosto) del 2002. Las plantas seleccionadas han sido cosechadas y las semillas sembradas en parcelas más grandes en comparación con el testigo local. Durante el 2003, las líneas promisorias seleccionadas de las alternativas FP-1 y FP-2 serán comparadas en ensayos replicados para estimar la efectividad de cada método; estos resultados serán presentados en otras publicaciones.

En la alternativa FC, la selección desde la F2 a la F6 fue realizada en el Zamorano sin participación de agricultores, representando el enfoque de mejoramiento convencional. Las selecciones se basaron en arquitectura de planta, resistencia a enfermedades (incluyendo al mosaico dorado amarillo, que no se presenta en Yorito), rendimiento y calidad de grano. Las evaluaciones en Zamorano, se condujeron con bajos niveles de insumos, incluyendo $65 \mathrm{~kg} / \mathrm{ha}$ de fórmula comercial 1846-0, de una a dos aplicaciones de insecticidas y sin aplicaciones de fungicidas. 
Es justo mencionar, que en la última década algunos programas de mejoramiento han venido incorporando diversos niveles de participación de agricultores; más aún, algunos programas están evolucionando hacia un aumento en los enfoques de FP. En los últimos años, estos enfoques participativos y el uso de una base genética más amplia, han contribuido con el incremento significativo de la adopción de cultivares de frijol en algunos países de Centroamérica.

\section{Ensayo comparativo de líneas avanzadas}

Un ensayo de adaptación y rendimiento para comparar las mejores líneas seleccionadas a través de las metodologías FP-1 vs. FC, denominado "ensayo comparativo", fue conducido durante la época de "postrera" (octubre-diciembre) del 2001. El ensayo consistió de 16 líneas, 10 F6 seleccionadas por agricultores de tres comunidades de Yorito (FP-1), cinco F7 seleccionada en Zamorano (FC) y la variedad criolla local, Concha Rosada, usada como testigo (Cuadro 1).

Cuadro 1. Líneas avanzadas incluidas en el ensayo comparativo del proceso de fitomejoramiento participativo de frijol en la región de Yorito. Honduras, 2001z.

\begin{tabular}{rlll}
\hline $\mathbf{N}^{\circ}$ & \multicolumn{1}{c}{ Código } & \multicolumn{1}{c}{ Identificación } & \multicolumn{1}{c}{ Procedencia } \\
\hline 1. & PPBY-1 & PPB 12-4-4-59M & Mina Honda \\
2. & PPBY-2 & PPB 11-98-2-5M & Chagüitío \\
3. & PPBY-3 & PPB 11-7-4-9M & Santa Cruz \\
4. & PPBY-4 & PPB 12-13-3-17M & Mina Honda \\
5. & PPBY-5 & PPB 11-60C & Zamorano \\
6. & PPBY-6 & PPB 12-68-4-5M & Mina Honda \\
7. & PPBY-7 & PPB 11-7-7-31M & Chagüitío \\
8. & PPBY-8 & PPB 11-44-5-13M & Santa Cruz \\
9. & PPBY-9 & PPB 11-88C & Zamorano \\
10. & PPBY-10 & PPB 12-68-3-56M & Mina Honda \\
11. & PPBY-11 & PPB 11-96C & Zamorano \\
12. & PPBY-12 & PPB 12-68-2-2M & Mina Honda \\
13. & PPBY-13 & PPB 12-28C & Zamorano \\
14. & PPBY-14 & PPB 11-7-10-33M & Chagüitío \\
15. & PPBY-15 & PPB 12-53C & Zamorano \\
16. & Concha Rosada & Testigo local & Región de Yorito \\
\hline
\end{tabular}

${ }^{\mathrm{z}}$ Ensayo conducido en cuatro localidades de Yorito y en el Zamorano durante la primera época del 2001. Las líneas fueron seleccionadas por los agricultores de las poblaciones PPB 11 y PPB 12 .

El ensayo fue conducido simultáneamente en cuatro comunidades de Yorito (Mina Honda, Chaguitío, Patastera y Santa Cruz) y en Zamorano. En cada localidad, se usó un diseño de bloques completos al azar con tres repeticiones. La unidad experimental fue surcos individuales de $5 \mathrm{~m}$ de largo; la distancia entre surcos fue de 0,6 m y las semillas se distribuyeron usando un espaciamiento de $10 \mathrm{~cm}$. Las aplicaciones de fertilizantes incluyeron $65 \mathrm{~kg} / \mathrm{ha}$ de $18-46-0$ a los 8 DDE y $65 \mathrm{~kg} / \mathrm{ha}$ urea a los 30 DDE. No se realizaron controles químicos de insectos o enfermedades.

Las líneas avanzadas fueron evaluadas por rendimiento de grano, días a floración y madurez, valor agronómico (índice de selección que incluye arquitectura de planta, carga reproductiva, reacción a enfermedades y días a madurez) y valor comercial del grano (incluyendo color, tamaño y forma). Una escala de 1 a $9(1=$ excelente y $9=$ muy pobre $)$ fue utilizada para cuantificar las diferencias entre las líneas. Adicionalmente al rendimiento, se estimaron los coeficientes de estabilidad de rendimiento de las líneas y el testigo, según Eberhart y Russell (1966). Las líneas fueron ordenadas por su rendimiento en cada localidad, para explicar su comportamiento en la misma localidad donde se seleccionaron y a través de localidades (interacción genotipo x ambiente). De este ensayo, los agricultores participantes decidieron seleccionar cuatro líneas (PPBY-2 y PPB-14 de Chaguitío, PPBY-1 de Mina Honda y PPBY-8 de Santa Cruz) para la fase siguiente de validación en parcelas de mayor tamaño.

\section{Ensayo de comprobación de variedades}

Los ensayos de validación del potencial de las líneas seleccionadas para convertirse en futuras variedades, conocidos en la región centroamericana como comprobación de variedades (COVA), fueron conducidos durante la época de primera del 2002. Estos ensayos incluyeron las cuatro líneas seleccionadas del ensayo comparativo mencionado anteriormente (PPBY-1, PPBY-2, PPBY-8 y PPBY-14), la variedad comercial “Tío Canela 75" y el testigo local Concha Rosada. El ensayo se condujo en las localidades de Mina Honda, Patastera y dos localidades de Santa Cruz), empleándose un diseño de bloques completos al azar con dos repeticiones por localidad; la unidad experimental estuvo constituida por parcelas de10 surcos distanciados a 50 $\mathrm{cm}$ y de $8 \mathrm{~m}$ de largo. Para la siembra se emplearon distanciamientos de $10 \mathrm{~cm}$ entre plantas. Las fertilizaciones fueron del orden de $65 \mathrm{~kg} / \mathrm{ha}$ de $18-46-0$ y $65 \mathrm{~kg}$ /ha de urea a los 8 y $30 \mathrm{DDE}$, respectivamente. No se efectuaron aplicaciones de pesticidas para el control de plagas. Las evaluaciones realizadas por los agricultores incluyeron el valor agronómico y la resistencia a la antracnosis (Colletotrichum lindemuthianum), mancha angular (Phaeoisariopsis griseola), oidium (Erysiphe poligony) y roya (Uromyces appendiculatus), en la etapa de llenado del grano; el rendimiento de grano por parcela a la cosecha y el valor comercial del grano a la postcosecha. La mejor línea de este ensayo de 
comprobación ha entrado a una fase de incremento de semilla bajo riego y estará siendo diseminada a través de las comunidades participantes y vecinas de la región de Yorito durante el presente año.

\section{RESULTADOS Y DISCUSIÓN}

\section{Evaluación de poblaciones de mejoramiento}

Los resultados que se incluyen en el documento fueron obtenidos con la participación de agricultores de Yorito. El proceso de selección desde generaciones tempranas (F3) a avanzadas (>F6), bajo la alternativa FP-1, fue iniciado durante la siembra de primera del 2000. Familias F3 de las poblaciones PPB 11, PPB 12, PPB 15 y PPB 16 fueron sembradas y evaluadas en Mina Honda, por agricultores de esta comunidad y de Chaguitío, Patastera y Santa Cruz. La selección fue basada en evaluaciones realizadas en las etapas de llenado de grano, cosecha y poscosecha. Las familias seleccionadas fueron avanzadas y evaluadas en las siguientes generaciones en cada comunidad; el número y las líneas seleccionadas variaron entre las comunidades. En la generación F3, los agricultores de Mina Honda y Santa Cruz aplicaron una alta intensidad de selección y sólo seleccionaron 15 de 120 líneas evaluadas (12,5\%); mientras que los agricultores de Patastera fueron más conservadores con respecto a mantener una "suficiente variabilidad para la siguientes selecciones" y seleccionaron un mayor número, 65 familias $(54,1 \%)$ (Cuadro 2). Como era predecible, los grupos de Mina Honda y Santa Cruz confrontaron los efectos de no tener suficiente diversidad para aplicar selección en la F4 y más adelante. Después de analizar los efectos del tamaño de las poblaciones, los agricultores concluyeron que una intensidad de selección de 30-40\% podría ser adecuada para mantener suficiente diversidad para continuar la selección. Como resultados de esta experiencia, la intensidad de selección en la generación F3 de las poblaciones PPB 15 y PPB 16, sembradas en la época siguiente, fue menos intensa y varió de 28 a $37 \%$ en tres comunidades (Mina Honda, Patastera y Santa Cruz).

En Patastera, los viveros de mejoramiento F6 de las poblaciones PPB 11 y PPB 12 se perdieron debido a lluvias excesivas durante la madurez fisiológica, en la época de primera del 2001. Una condición de alta humedad representa un problema serio durante la época de cosecha, porque los agricultores cosechan las plantas de frijol y las dejan en manojos a secar en el campo esperando una condición climática favorable (seca y soleada). Algunos agricultores cosechan sus frijoles y los cuelgan bajo áreas cubiertas facilitando su secado. Bajo estas condiciones, a veces la calidad de la semilla
Cuadro 2. Secuencia de selección de familias de cuatro poblaciones segregantes de frijol (derivadas de cruzas criolla $\mathrm{x}$ élite) usando métodos de fitomejoramiento participativo. Yorito, Honduras, 2000-2002.

\begin{tabular}{lcccr}
\hline \multirow{2}{*}{ Comunidad } & \multicolumn{4}{c}{ Familias seleccionadas por generación } \\
\cline { 2 - 5 } & F3 & F4 & F5 & F6 \\
\hline & \multicolumn{4}{c}{ Poblaciones PPB 11 y PPB 12z } \\
\cline { 3 - 5 } Mina Honda & \multirow{2}{*}{120} & 15 & 65 & 5 \\
Chagüitío & & 22 & 33 & 3 \\
Santa Cruz & & 15 & 24 & 2 \\
Patastera & & 65 & 80 & perdido $^{y}$
\end{tabular}

Poblaciones PPB 15 y PPB 16

$\begin{array}{lllll}\text { Mina Honda } & 107 & 40 & 15 & 5 \\ \text { Patastera } & & 35 & 12 & 4 \\ \text { Santa Cruz } & & 30 & 25 & 4\end{array}$

${ }^{\mathrm{z}}$ Las evaluaciones de PPB 11 y PPB 12 se iniciaron en la primera época, y las de PPB 15 y PPB 16 en la segunda época del 2000. y lluvias excesivas durante la madurez causaron daños y germinación prematura de la semilla antes de la cosecha.

es severamente afectada por las lluvias o alta humedad ambiental las cuales pueden causar infecciones secundarias por microorganismos y germinación prematura.

\section{Ensayo comparativo de líneas avanzadas}

Los resultados del ensayo comparativo conducido en la segunda época de siembra del 2001, que incluyó 10 líneas seleccionadas mediante FP en Yorito, cinco líneas de Zamorano seleccionadas por FC y el testigo local Concha Rosada, se presentan en el Cuadro 3. Los mejores rendimientos promedios fueron obtenidos principalmente en las líneas FC seleccionadas por mejoramiento convencional; aún más, tres de las cinco líneas seleccionadas en Zamorano estuvieron entre las cinco líneas de mayor rendimiento, posiblemente debido a un mayor rendimiento promedio en esta localidad.

Es interesante que la línea de Zamorano PPBY-13 fue la mejor en este lugar; mientras que, la línea de Chagüitío PPBY-7 fue la mejor en Mina Honda y Santa Cruz, y la segunda en Patastera, pero resultó en octavo lugar en Chagüitío, la comunidad donde fue desarrollada. Es necesario señalar que los rendimientos de la localidad de Chagüitío fueron los más bajos debido a las condiciones de sequía durante el ensayo. En general, las mejores líneas de Chagüitío, Mina Honda y Zamorano, se comportaron muy bien en sus propias localidades; sin embargo, las dos líneas seleccionadas en Santa 
Cuadro 3. Rendimiento de grano, coeficientes de estabilidad y posición por rendimiento en las comunidades donde se condujeron los ensayos. Ensayo comparativo de líneas avanzadas de frijol, Yorito, Honduras, 2001.

\begin{tabular}{|c|c|c|c|c|c|c|c|c|c|}
\hline \multirow{2}{*}{\multicolumn{2}{|c|}{ Línea (procedencia) ${ }^{\mathrm{Z}}$}} & \multirow{2}{*}{$\begin{array}{c}\text { Rendimiento } \\
(\mathrm{kg} / \mathrm{ha})\end{array}$} & \multicolumn{2}{|c|}{ Coef. Estabil. $^{\mathbf{y}}$} & \multicolumn{5}{|c|}{ Posición/comunidad } \\
\hline & & & $b$ & $S b$ & $\mathbf{Z A}$ & $\mathbf{C H}$ & MH & SC & PT \\
\hline PPBY-13 (ZA) & & 1.459 & 1,46 & 0,18 & 1 & 4 & 6 & 3 & 3 \\
\hline PPBY-7 (CH) & & 1.403 & 1,07 & 0,22 & 5 & 8 & 1 & 1 & 2 \\
\hline PPBY-2 (CH) & - & 1.306 & 1,43 & 0,22 & 2 & 9 & 12 & 4 & 15 \\
\hline PPBY-11 (ZA) & & 1.245 & 0,89 & 0,20 & 13 & 5 & 5 & 2 & 5 \\
\hline PPBY-9 (ZA) & & 1.147 & 1,11 & 0,28 & 4 & 3 & 4 & 16 & 14 \\
\hline PPBY-6 (MH) & & 1.143 & 0,81 & 0,19 & 14 & 7 & 2 & 13 & 1 \\
\hline C. Rosada (testigo) & & 1.126 & 0,71 & 0,23 & 16 & 2 & 3 & 6 & 11 \\
\hline PPBY-4 (MH) & & 1.125 & 0,82 & 0,04 & 11 & 1 & 8 & 9 & 10 \\
\hline PPBY-15 (ZA) & & 1.124 & 1,19 & 0,23 & 3 & 12 & 16 & 10 & 8 \\
\hline PPBY-3 (SC) & & 1.106 & 0,95 & 0,06 & 9 & 14 & 9 & 7 & 7 \\
\hline PPBY-12 (MH) & & 1.106 & 1,04 & 0,12 & 6 & 13 & 14 & 5 & 13 \\
\hline PPBY-10 (MH) & & 1.088 & 0,93 & 0,06 & 10 & 15 & 10 & 8 & 6 \\
\hline PPBY-8 (SC) & - & 1.071 & 0,92 & 0,08 & 8 & 11 & 11 & 12 & 4 \\
\hline PPBY-14 (CH) & - & 1.015 & 0,92 & 0,12 & 7 & 6 & 15 & 14 & 12 \\
\hline PPBY-5 (ZA) & & 1.015 & 0,89 & 0,10 & 12 & 16 & 7 & 15 & 9 \\
\hline PPBY-1 (MH) & - & 930 & 0,84 & 0,05 & 15 & 10 & 13 & 11 & 16 \\
\hline Promedio (kg/ha) & & 1.151 & & & 2,125 & 270 & 1,319 & 1,377 & 661 \\
\hline DMS $(0,10)$ & & ns & & & 824 & ns & 552 & 612 & 301 \\
\hline
\end{tabular}

z ZA (Zamorano), CH (Chagüitío), MH (Mina Honda), SC (Santa Cruz) and PT (Patastera).

y Eberhart and Russell (1966).

- Líneas seleccionadas para posterior validación en ensayos de comprobación.

Cruz fueron pobres en esta localidad (7ma. y 12va.), y las mejores líneas fueron de Chagüitío y de Zamorano. Los coeficientes de estabilidad de rendimiento (donde $\mathrm{b}=1 \mathrm{y} \mathrm{Sb}=0$, indican la mayor estabilidad) sugieren que la línea de mejor rendimiento y estabilidad fue PPBY-7 de Chagüitío. De acuerdo con el análisis de estabilidad, las líneas PPBY-13 y PPBY-2 rindieron mejor bajo condiciones favorables; y las líneas PPBY-11 y PPBY- 6 bajo condiciones menos favorables. Los valores altos del coeficiente $\mathrm{Sb}$ sugieren efectos ambientales significativos y la necesidad de un mayor número de localidades y repeticiones para incrementar la eficiencia experimental de este tipo de ensayos.

No se observaron diferencias significativas en los días a floración y madurez, valor agronómico y valor comercial del grano (Cuadro 4). Lo anterior sugiere que fue posible la selección de líneas de frijol con algunas características similares, a pesar de que las selecciones se llevaron a cabo en diferentes localidades; atribuyéndose las diferencias observadas a las contribuciones de los progenitores.

Como se esperaba, las líneas seleccionadas en Zamorano fueron las únicas que presentaron resistencia ( severidad $<3$ ) en esta localidad, al presentarse una presión moderada del virus del mosaico dorado amarillo; las líneas restantes, fueron intermedias o susceptibles
Cuadro 4. Días a floración y madurez, valor agronómico, valor comercial del grano y resistencia a cinco enfermedades. Ensayo comparativo de líneas avanzadas de frijol, Yorito, $2001^{\mathrm{Z}}$.

\begin{tabular}{lcccccccccc}
\hline \multicolumn{1}{c}{ Línea } & DF & DM & VA & VC & MD & AN & MA & MP RY \\
\hline PPBY-13 & 50 & 92 & 5.2 & 5,4 & R & I & I & S & R \\
PPBY-7 & 53 & 93 & 5,7 & 5,3 & I & I & I & I & I \\
PPBY-2 & 54 & 93 & 5,3 & 5,5 & I & I & I & S & I \\
PPBY-11 & 50 & 91 & 5,5 & 3,8 & S & I & I & I & I \\
PPBY-9 & 51 & 90 & 5,2 & 5,2 & I & R & I & R & R \\
PPBY-6 & 50 & 91 & 4,7 & 4,0 & S & I & I & S & R \\
C. Rosada (testigo) & 51 & 89 & 5,5 & 3,5 & S & S & S & I & I \\
PPBY-4 & 50 & 88 & 4,3 & 5,4 & S & I & I & I & I \\
PPBY-15 & 51 & 92 & 5,5 & 4,2 & I & R & I & I & R \\
PPBY-3 & 52 & 93 & 6,0 & 3,5 & S & I & I & I & I \\
PPBY-12 & 52 & 93 & 5,8 & 4,7 & I & I & I & I & I \\
PPBY-10 & 51 & 91 & 5,3 & 4,8 & I & I & I & S & I \\
PPBY-8 & 51 & 90 & 4,7 & 4,0 & S & R & I & R & R \\
PPBY-14 & 51 & 94 & 5,8 & 4,2 & S & I & I & I & R \\
PPBY-5 & 52 & 92 & 5,0 & 4,4 & R & I & S & S & R \\
PPBY-1 & 43 & 92 & 5,0 & 4,0 & S & I & I & I & \\
RDMS (0,10) & ns & ns & ns & ns & & & & & \\
\hline
\end{tabular}

z DF (días a floración), DM (días a madurez), VA (valor agronómico) y VC (valor comercial del grano), promedio de cinco localidades; MD (mosaico dorado amarillo) en Zamorano; y AN (antracnosis), MA (mancha angular), MP (mildiú polvoso u oidium) y RY (roya), promedio de cuatro localidades de Yorito.

S (Susceptible), I (intermedia) y R (resistente).

- Líneas seleccionadas para posterior validación en ensayos de comprobación. 
(Cuadro 4). Esto fue posible, debido a que las líneas élites usadas en los cruzamientos con la variedad criolla Concha rosada, son resistentes a esta enfermedad viral. Para las enfermedades observadas en las cuatro localidades de Yorito, la región meta, las líneas PPBY-11 de Zamorano y PPBY- 8 de Santa Cruz, presentaron las mejores combinaciones de resistencia a la antracnosis, mancha angular, oidium y roya.

Una vez procesada la información y revisados los resultados del ensayo comparativo durante un taller, cuatro líneas de Yorito ( PPBY-2 y PPBY-14 de Chagüitío, PPBY-1 de Mina Honda y PPBY-8 de Santa Cruz) fueron escogidas por los agricultores para su evaluación posterior. Se decidió validar su potencial como variedades en un ensayo de comprobación de variedades (COVA), que se describe más adelante. Según los datos de rendimiento, es posible argumentar que de las cuatro líneas seleccionadas para validación sólo la línea PPBY-2 se comportó bien (tercera posición) en el ensayo comparativo; las otras tres líneas ocuparon las posiciones más bajas y tuvieron un rendimiento inferior al testigo (Cuadro 3). A pesar de esto, estas cuatro líneas fueron escogidas por los agricultores para el ensayo de comprobación de variedades, con base a otros atributos incluyendo arquitectura, precocidad, resistencia a enfermedades y calidad de grano, considerados con caracteres importantes para ellos.

Está claro que un buen número de ensayos y épocas son necesarios para obtener información más precisa que permita determinar los beneficios de seleccionar líneas bajo condiciones específicas de regiones meta, como Yorito, donde frecuentemente se presentan variaciones sustanciales de suelo y clima, así como diferencias en las prácticas de manejo del cultivo. Aún así estos resultados no descartan la posibilidad de desarrollar líneas avanzadas útiles para pequeños productores por métodos convencionales, siempre y cuando un número considerable de líneas homocigotas, pero diversas, son evaluadas por adaptación específica bajo condiciones locales. Otros factores agronómicos y socioeconómicos deberán también ser tomados en cuenta.

Las experiencias sugieren que los agricultores necesitan desarrollar alternativas para mantener bajo buenas condiciones de almacenamiento (a corto y mediano plazo), la semilla remanente de las líneas durante el proceso de FP, para asegurar que estas pueden ser reemplazadas en caso de pérdida por eventos climáticos, como condiciones de sequía y lluvias excesivas, que pueden destruir juegos completos de germoplasma mejorado en el campo. Durante los tres años de actividades bajo este proyecto, se perdió semilla de poblaciones completas en dos oportunidades, debido a sequía y a lluvias excesivas; afortunadamente, estos materiales fueron repuestos con semilla remanente mantenida bajo condiciones de bajas temperaturas en el banco de germoplasma del Zamorano.

\section{Ensayo de comprobación de variedades}

Las cuatro líneas seleccionadas en el ensayo comparativo, PPB-1, PPBY-2, PPBY-8 y PPBY-14, y dos testigos comerciales, Concha Rosada y Tío Canela 75 (la variedad criolla y mejorada más cultivadas en la región de Yorito), fueron evaluadas en cuatro localidades de Yorito (Mina Honda, Patastera y dos de Santa Cruz) durante la primera del 2002. Los resultados de las evaluaciones de rendimiento, días a floración y madurez, resistencia a enfermedades y valor comercial del grano se presentan en el Cuadro 5.

Cuadro 5. Rendimiento de grano, días a floración y madurez, valor comercial del grano y resistencia a enfermedades. Ensayo de comprobación de variedades de frijol, Yorito, Honduras, 2002.

\begin{tabular}{lcccccccc}
\hline \multicolumn{1}{c}{ Línea } & $\begin{array}{c}\text { Rdto. } \\
(\mathbf{k g} / \mathbf{h a})\end{array}$ & DF & DM & VC & AN & MA & MP RY \\
& 2.123 & 40 & 72 & 2,5 & 3,3 & 5,0 & 3,3 & 2,8 \\
\hline PPBY-14 & 2.012 & 40 & 72 & 2,5 & 3,8 & 6,3 & 3,0 & 3,0 \\
PPBY-8 & 1.915 & 42 & 78 & 3,0 & 3,3, & 4,5 & 3,0 & 3,5 \\
PPBY-2 & 1.759 & 42 & 72 & 1,5 & 3,3 & 5,3 & 3,0 & 4,3 \\
PPBY-1 & 1.646 & 39 & 70 & 3,0 & 5,5 & 6,0 & 4,0 & 5,3 \\
C. Rosada (testigo) \\
Tío Canela 75 & 1.461 & 42 & 78 & 3,5 & 4,3 & 5,0 & 3,0 & 4,5 \\
& & & & & & & & \\
DMS (0,05) & 572 & ns & 5,2 & 1,0 & 1,0 & 1,2 & 0,8 & 1,1
\end{tabular}

z DF (días a floración), DM (días a madurez), VC (valor comercial del grano), AN (antracnosis), MA (mancha angular), MP (mildiú polvoso u oidium) y RY (roya). Promedio de cuatro localidades.

La línea PPBY-14 presentó el mayor rendimiento de grano, pero sólo superó a los testigos comerciales; estadísticamente $(\mathrm{P}<.10)$, las cuatro líneas seleccionadas mediante FP fueron iguales. Por otro lado, con excepción de la línea PPBY-2, que resultó ser más tardía, las demás presentaron la floración y madurez ligeramente después (2-3 días) que la variedad criolla Concha Rosada. Usando las evaluaciones del valor comercial de grano de Patastera y Mina Honda, la mejor línea fue PPBY-1; las otras tres líneas fueron ligeramente de mejor calidad (color, forma y tamaño) que la local Concha Rosada y superior a Tío Canela 75. Se observaron contrastes en los valores asignados al valor comercial de las líneas PPBY-8 y PPBY-14; la primera línea fue evaluada superior y la segunda inferior a Concha Rosada en la localidad de Patastera, y lo opuesto para ambas líneas en la comunidad de Mina Honda. 
Como se ha observado anteriormente en muchas regiones de Honduras y de Centroamérica, que producen y consumen grano rojo pequeño (raza Mesoamericana), las preferencias de grano de los agricultores pueden variar aún entre comunidades relativamente cercanas; esto sugiere, que se deberá ofrecer suficiente diversidad en cuanto a la característica de grano rojo pequeño, en las poblaciones segregantes y líneas avanzadas que se faciliten a los agricultores a través de procesos convencionales o participativos.

\section{CONCLUSIONES Y RECOMENDACIONES}

Después de tres años de actividades de investigación y entrenamiento en FP, los agricultores de Yorito y del Lago Yojoa involucrados, han adquirido un nivel deseable de conocimientos y destrezas para desarrollar variedades mejoradas para sus condiciones locales. Actualmente, son capaces de manejar viveros de mejoramiento y ensayos de líneas avanzadas siguiendo principios básicos de diseños experimentales. También son capaces de evaluar poblaciones segregantes y líneas avanzadas en etapas críticas de desarrollo, incluyendo el llenado de vainas, cosecha y postcosecha, así como seleccionar simultáneamente por varios caracteres. Estos agricultores han seguido procesos de selección desde generaciones tempranas (familias F3) hasta pruebas de líneas avanzadas, incluyendo ensayos comparativos (parcelas de surcos individuales) y validaciones (parcelas grandes de varios surcos). Los agricultores de estas regiones han estado involucrados en procesos de diseminación de cultivares que fueron desarrollados a través de actividades de investigación participativa; y actualmente están en proceso de hacerlo con las líneas promisorias seleccionadas a través de FP.

Como resultado de actividades de investigación participativa, llevadas a cabo antes del inicio de las actividades de FP, y que fueron continuadas bajo este proyecto de FP como parte de la fase de actividades de validación y diseminación de variedades mejoradas, las líneas promisorias DICZA 9801 y PRF 9707-36 (nombre varietal "Palmichal 1") fueron seleccionadas para su difusión como variedades mejoradas locales por los CIALes participantes del Lago Yojoa. En Yorito, se seleccionaron las líneas PTC 9557-10 y PRF 9633-11 (nombre varietal "Cayetana 85") para su validación y diseminación en dos comunidades participantes. Aunque todavía no se ha logrado en ambas regiones alcanzar un nivel de diseminación deseado, varios agricultores las han adoptado como variedades y mediante su cultivo se están beneficiando de las ventajas agronómicas con relación a las variedades criollas tradicionales.
El ensayo comparativo sugirió que adicionalmente al buen rendimiento, los agricultores seleccionan líneas promisorias por otros caracteres deseables como arquitectura erecta, maduración temprana, resistencia a enfermedades y calidad de grano. Como se mencionó anteriormente, las cuatro líneas seleccionadas de este ensayo comparativo para su posterior validación en los ensayos COVA, no fueron necesariamente las de mayor rendimiento; algunas fueron escogidas por la resistencia combinada a la antracnosis, mancha angular, oidium y roya, que son las enfermedades predominantes en la región. Sin embargo, la línea que presentó la mejor resistencia múltiple, PPBY-9, no fue seleccionada por presentar muy pobre rendimiento en Santa Cruz y $\mathrm{Pa}-$ tastera; esta línea tuvo un rendimiento muy bueno en las otras tres localidades, pero demostró falta de estabilidad. Esto sugiere que los agricultores pudieron usar con eficiencia los resultados obtenidos de varias localidade, para seleccionar por factores múltiples incluyendo estabilidad de rendimiento. Es obvio que a veces es difícil entender la complejidad de análisis que emplean los agricultores para tomar decisiones. Por lo cual, el incremento de las interacciones con los agricultores es fundamental para lograr mayor adopción de variedades mejoradas.

Nuestro programa continuará involucrado en actividades de FP con agricultores de Yorito y el Lago Yojoa. Por los próximos años, líneas avanzadas y poblaciones segregantes serán facilitadas a estos grupos de agricultores, a fin de ampliar el mejoramiento de las variedades criollas y dar acceso a germoplasma mejorado. Con base a estas experiencias en FP, se argumenta la necesidad de desarrollar métodos simplificados que involucren a los agricultores y organizaciones de desarrollo en procesos de FP a una mayor escala. Se debe preveer que estos métodos observen los principios y prácticas básicas de la genética, fitomejoramiento y diseños experimentales; además, las metodologías deben ser desarrolladas teniendo en mente un rol activo de los agricultores y sus realidades agrícolas y socioeconómicas.

Padres donantes que poseen genes de resistencia a razas específicas de los patógenos predominantes en las áreas metas, y genes que incrementen la adaptación a factores abióticos limitantes de la producción (p.e. sequía y baja fertilidad), así como el incremento en el contenido y disponibilidad de nutrientes (p.e. hierro y zinc), deben de ser incorporados en las variedades de los agricultores mediante hibridación. La selección asistida con marcadores debe de ser usada en las pruebas de generaciones tempranas de poblaciones de mejoramiento, a ser manipuladas por los agricultores bajo enfoques de FP, a fin de incrementar las posibilidades de desarrollar cultivares útiles para condiciones locales. El entrenamiento de técnicos y agricultores involucrados en estas actividades continuará 
siendo un componente importante para la aplicación exitosa de métodos de FP.

Por otro lado, los fitomejoradores pueden sacar ventajas de verse involucrados en actividades de FP y obtener algunos beneficios de estos métodos, como la gran oportunidad de utilizar localidades específicas para evaluación de germoplasma y la colaboración de socios que combinan experiencia en la conducción de ensayos de campo con el conocimiento local, los cuales muchas veces son difíciles de tener en programas convencionales. Bajo métodos de FP, los agricultores tienen mayor oportunidad de probar líneas mejoradas bajo sus propias condiciones; esto podría representar un camino más rápido y efectivo para incrementar la adopción de germoplasma mejorado.

\section{RECONOCIMIENTOS}

El apoyo para la conducción de estas actividades de mejoramiento participativo en frijol en Honduras, durante el período de 1999-2002, fue proporcionado por el Programa de Small Grants del PRGA/CGIAR. Actualmente, estas actividades son apoyadas por el Bean/Cowpea CRSP (Donación USAID No. GDG-G- 00-0200012-00) y el Fondo Noruego para el Desarrollo.

\section{LITERATURA CITADA}

ASHBY, J.A.; GRACIA, T.; GUERRERO, M.P.; QUIRÓS, C.A.; ROA, J.I.; BELTRÁN, J.A. 1995. Institutionalising farmer participation in adaptive technology testing with the CIAL. Agricultural Research and Extension Network 57 (Londres): 43 p.

COTTY, D.; GARCÍA, M.; ESTRADA, I. ; ANCHUNDIA, E. 2001. Indicadores básicos sobre el desempeño agropecuario 1971-2000. Proyecto de Información Agrícola y Análisis de Políticas, Zamorano/USAID. Zamorano, Honduras, 110 p.

EBERHART, S.A. ; RUSSELL, W.A. 1966. Stability parameters for comparing varieties. Crop Sc. 6:36-40.

HUMPHRIES, S.; GONZALES, J.; JIMÉNEZ, J.; SIERRA, F. 2000. Searching for sustainable land use practices in Honduras: lessons from a programme of participatory research with hillside farmers. Agricultural Research and Extension Network 104 (Londres): 16 p.

ROSAS, J.C.; CASTRO, A.; FLORES, E. 2000. Mejoramiento genético de frijol rojo y negro mesoamericano para Centroamérica y El Caribe. Agronomía Mesoamericana 11(2): 37-46.

ROSAS, J.C. 2001. Aplicación de metodologías participativas para el mejoramiento genético de frijol en Honduras. Agronomía Mesoamericana 12(2): 219-228. 